\title{
Teaching pigeons an abstract relational rule: Insideness
}

\author{
R. J. HERRNSTEIN, W. VAUGHAN, JR., D. B. MUMFORD, and S. M. KOSSLYN \\ Harvard University, Cambridge, Massachusetts
}

\begin{abstract}
In 49 sessions, pigeons failed to learn to sort a collection of 80 stimuli composed of a closed curve and a dot, divided into two categories, according to whether the dot was or was not inside the curve. Next, the pigeons were successfully trained, first with the insides of the curves shown in bright red, then with a darker red, and finally with a black matching the background outside the curve. After this stepwise procedure, the pigeons displayed a limited ability to sort novel curves and dot locations according to whether the dot was or was not inside the curve.
\end{abstract}

Pigeons learn rapidly and well to identify complex patterns and representations of objects in two-dimensional visual scenes containing people, bodies of water, trees, letters of the alphabet, silhouettes of oak leaves, and so on (see review in Herrnstein, 1984, and several of the chapters in Commons, Herrnstein, \& Wagner, 1983). On the other hand, pigeons learn only with difficulty, if at all, the general rule that would enable them to signal whether or not an arbitrary pair of visual stimuli are identical to each other or, if given a target, to pick the odd or matching stimulus out of any arbitrary set (e.g., Pisacreta, Lefave, Lesneski, \& Potter, 1985; Urcuioli, 1985; Zentall \& Hogan, 1978). Some researchers (e.g., Mackintosh, 1983) believe that pigeons (and presumably other subprimate species) are incapable of mastering such abstract relational rules at all. The weak or marginal evidence of relational discrimination reported by some researchers is then dismissed as artifactual.

Easy success with one kind of discrimination problem and difficulty or failure with the other is a riddle that may somehow be solved by analysis of the notion of relationality (Herrnstein, 1985). The riddle is well known but not yet solved. According to, for example, feature-based theories of recognition (e.g., Lindsay \& Norman, 1977), to identify a tree, the pigeon needs a set of descriptors based on local properties of the stimulus, which may be complex but not necessarily relational in any abstract sense, and then must aggregate the evidence for "tree-ness" from each descriptor. But, to master a general oddity or matching rule, aggregating the evidence from a set of descriptors of the stimuli per se will not suffice. To learn a general, or abstract, relational rule, what is needed, in addition to descriptors of the stimuli being interrelated, is some sort of predication linking the stimuli that does not include descriptive features of the stimuli taken in-

The work reported here was supported by Grant IST-85-11606 from the National Science Foundation to Harvard University. Correspondence may be sent to R. J. Herrnstein, Department of Psychology, Harvard University, William James Hall, 33 Kirkland St., Cambridge, MA 02138. dividually. The distinction is between being able to detect that stimuli contain prescribed features, resemble particular exemplars, or satisfy certain constraints on size, color, aspect ratio, and so forth versus, on the other, being able to detect that they conform to an abstract relation in general, such as "equal to," "above," or the like. Description may be complex without limits, but still fall short of predication as defined here. Lower animals evidently have greater difficulty with description plus predication than they do with mere description.

The distinction being made here resembles one suggested by Ullman (1984) in a discussion of visual routines. Ullman is concerned, not with subhuman visual discrimination, but with the computations implicit in human vision and their implications for how the nervous system works and for building a vision machine. He concludes that certain obvious features of performance imply that there must be, in addition to representations arising from parallel, local, and bottom-up computations like those embodied in, for example, Marr's (1976) primal sketch-a "top-down application of visual routines to the representations constructed in the first stage" (Ullman, 1984, p. 84f). Visual routines are implied because the human visual system readily accomplishes feats of discrimination that would greatly tax a purely bottom-up representation of the visual field, feature by feature. A visual routine in Ullman's sense resembles what we referred to above as an abstract relational rule, that is to say, a predication among descriptors.

Consider, Ullman suggests, our capacity to decide whether or not an element in the visual field is inside a defined region-for example, whether or not a dot is inside a closed curve. This particular relation is known to pose a computational challenge to some feature-based models of vision (e.g., Minsky \& Papert, 1969). Nothing about the local geometry of curve and dot can reliably identify insideness, since the curve may have bays or bulges that create concavities or convexities in the vicinity of the dot, independent of insideness. Yet the problem is trivially simple for us, as long as the region itself is not so convoluted that we fail to identify it as a region. 
This, says Ullman, is the result of a visual routine that tests for insideness, a top-down computation on the bottom-up representation of curve and dot. He suggests it may be something akin to "coloring" the interior of the curve mentally, then noting whether or not the dot falls on the color. Other routines may be imagined, such as mentally guiding the dot to see if it can be moved clearly away from the interior of the curve without crossing the curve.

Whether one's goal is to understand the neurophysiology of vision or to build a vision machine, it would be useful to know whether given functions are realized in simpler organisms than human beings. In the present study, we attempt to determine whether pigeons are capable of using, or being taught to use, the relation of insideness. If they can, then we would know that nervous systems substantially simpler than that of the human can be "programmed" to use a visual routine of considerable subtlety. And if they cannot, then the nature of their deficiencies may shed light on how, and to what extent, a visual system lacking the capacity for an abstract relation deals with an environment designed to make the use of that relation adaptive, such as the one created by the present procedure.

\section{METHOD}

\section{Subjects}

The subjects were 4 experimentally naive, white Carneaux pigeons. They were run at approximately $80 \%$ of free-feeding weights.

\begin{abstract}
Apparatus
A standard pigeon chamber was modified to allow the backprojection of $35-\mathrm{mm}$ slides onto a centered screen key, $.044 \mathrm{~m}$ high $x .064 \mathrm{~m}$ wide. To the left of the screen was a standard pigeon key, and below that was a hopper for the delivery of mixed grain. Reinforcement consisted of 2-sec access to the grain (4 sec at the beginning of each session). Slides were presented by a Mast System 2 random access projector, which could show any of 80 slides, based on signals over an RS-232 line from a Digital PDP 11/73 computer that controlled experimental events and collected data.
\end{abstract}

\section{Procedure}

Following training to peck the screen key and the left key, the pigeons started on the experiment proper. At the beginning of each session, the left key was illuminated with a red light, and the chamber was generally illuminated with a white light. A single peck to the red key turned off the keylight and the chamber light and produced reinforcement for $4 \mathrm{sec}$. At the end of reinforcement, the chamber light came on, and, $6 \mathrm{sec}$ later, the first slide was shown. The keylight remained out and the left key remained inactive for the rest of the session.

During the first $10 \mathrm{sec}$ of the slide presentation, responses to the slide key were recorded but had no other consequence. Following that, a variable-interval schedule of reinforcement averaging $10 \mathrm{sec}$ (VI $10 \mathrm{sec}$ ) began. At the end of an interval in the schedule, if the slide was a positive (defined below), a response within $2 \mathrm{sec}$ of another response produced $2 \mathrm{sec}$ of reinforcement, during which time the chamber light was off and the slide remained on. At the end of reinforcement, the slide went off, the chamber light came on, and, $6 \mathrm{sec}$ later, the next slide was shown. On Session 11, since no discrimination was evident, the time between responses required for reinforcement was reduced from 2 to $1 \mathrm{sec}$.
If the slide was a negative, after an interval in the VI $10 \mathrm{sec}$ finished and $5 \mathrm{sec}$ elapsed without a peck, the slide went off and, $6 \mathrm{sec}$ later, the next slide was shown. On Session 11, the program was changed to require $10 \mathrm{sec}$ without responding before a slide would go off.

The 80 slides in the projector tray were all different. For the slides used initially, there was a black closed curve on a contrasting background with a black dot either inside or outside (illustrated in Figure 1). For Pigeons 1 and 2, curves with dots inside were positive and those with dots outside were negative; the opposite held for Pigeons 3 and 4 . Since some of the curves protruded inward. and some outward, it would be difficult for the pigeon to tell if a dot near the protrusion was inside or outside by looking just in the vicinity of the dot. The order of the slides was random, with the constraints that no more than four dot-inside stimuli or four dot-outside stimuli could occur in a row and that each of the 10 groups of eight slides contained four of each kind. The order was changed each day, but was the same for all 4 birds on a given day. Each session ended after all $\mathbf{8 0}$ slides in the slide tray had been seen. During the course of the experiment, the stimuli were changed in various ways. These changes are described in sequence in the Results section that follows.

\section{RESULTS}

After 49 sessions on the basic procedure, using the same 80 slides every session, none of the 4 pigeons showed any consistent or appreciable discrimination between positive and negative stimuli. The left column of Table 1 gives the median relative responding to positives [i.e., positive responses/(positive + negative responses), or $\mathrm{P} /(\mathrm{P}+\mathrm{N})]$ for Sessions 45 to 49 . Although each pigeon responded slightly more to positive stimuli than to negative, the level of discrimination was negligible by the standards of this

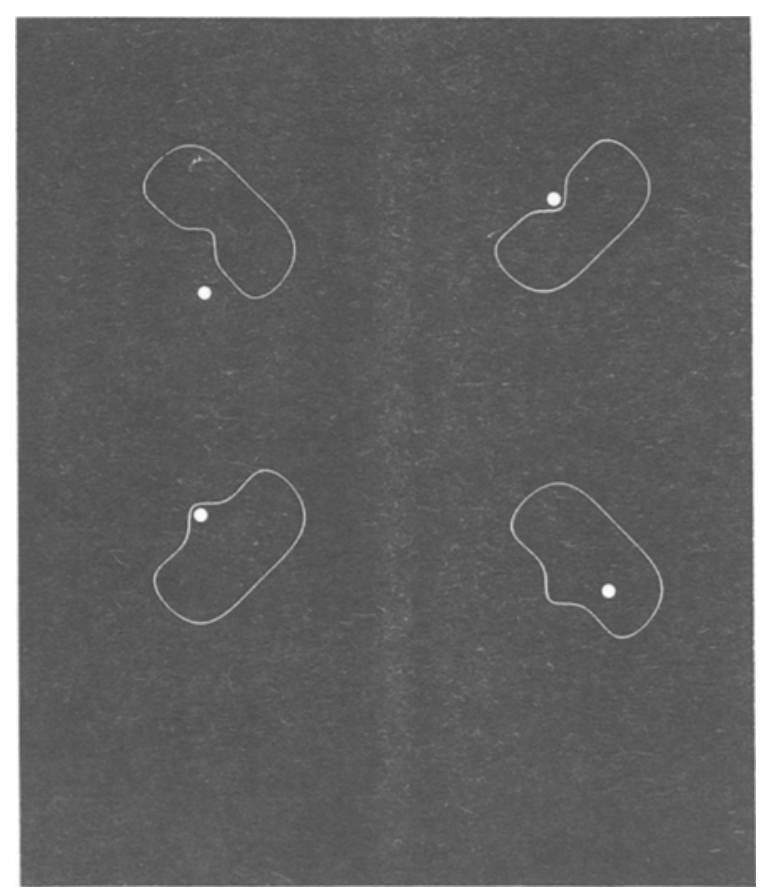

Figure 1. Four typical stimuli, illustrating dot-inside and dotoutside, and bays and bulges in the closed curve. Orientation and location of the closed curve varied, as shown here. 
Table 1

Relative Responding to Positive Stimuli

\begin{tabular}{cccc}
\hline & & \multicolumn{2}{c}{ Sessions 51-52 } \\
\cline { 3 - 4 } Subject & $\begin{array}{c}\text { Sessions } \\
45-49\end{array}$ & $\begin{array}{c}\text { Green } \\
\text { Test }\end{array}$ & $\begin{array}{c}\text { Control for } \\
\text { Green Test }\end{array}$ \\
\hline \multicolumn{5}{c}{$\begin{array}{c}\text { Dot-Inside } \\
1\end{array}$} & .52 & .59 & .54 \\
2 & .57 & .68 & .68 \\
& & Dot-Outside & \\
3 & .51 & 1.00 & .51 \\
4 & .51 & .99 & .50 \\
\hline
\end{tabular}

procedure using other classes of visual stimuli (e.g., Herrnstein \& de Villiers, 1980; Vaughan \& Greene, 1984).

Since previous research (e.g., Herrnstein \& de Villiers, 1980) has indicated that pigeons can learn, with a high degree of accuracy and within a dozen or so sessions, even an arbitrary division of $\mathbf{8 0}$ slides of natural scenes into two categories, there was some question about the virtual failure to discriminate in our experiment. That is, even if the pigeons were unable to use the inside-outside rule, previous work suggests that they might have been able, by rote, to memorize which slides were positive and which were negative. Consequently, three sessions were here interposed, in which half of the slides with dots inside were covered by a transparent green filter. The results of the latter two of these three "green test" sessions are given in the two columns of Table 1 under Sessions 51-52. Relative responding [i.e., $P /(P+N)]$ to stimuli with dots when they were covered with a green filter is shown under Green Test; for stimuli with dots not so covered, during the same sessions, relative responding is under Control for Green Test. Note that for Pigeons 1 and 2 , the green stimuli are positive, but that for Pigeons 3 and 4 , they are negative.

All pigeons increased in their proportions of correct responding, but Pigeons 3 and 4 especially did so during the green test. It is well established in discrimination learning that stimulus novelty is likely to inhibit responding. Any such tendency for responding to be suppressed by stimulus novelty thus worked to the advantage of Pigeons 3 and 4 and to the disadvantage of Pigeons 1 and 2 . The green test nevertheless indicates that the procedure is capable of producing discrimination among slides, and it further suggests (in light of earlier work cited above) that the failure to discriminate during the first $\mathbf{4 5}$ sessions was due to the nature of the stimuli themselves rather than the discrimination procedure. Not only did the pigeons fail to use insideness as a rule for discriminating, but the stimuli posed a difficult problem in rote memory.

\section{Phase 1}

Starting with Session 53, the green filter was removed and the stimuli were modified in an attempt to teach the pigeons the concept of insideness directly. These procedural modifications are outlined in Table 2, along with the numbers of sessions they were in force. With the exception of Procedure 5 (see below), Phase $1 \mathrm{em}$ ployed the same 80 slides as in the earlier sessions, changing only the colors of various elements. The exterior of the curve was now black, the curve and the dot were white. What varied in most of Phase 1 (except as indicated below) was the color of the interior of the curve.

It is convenient to think of the 80 slides as two 40 -slide half-trays, each with 20 dot-inside and 20 dot-outside slides in it. The apparatus, in fact, sampled at random from the slide tray as a session proceeded, but our experimental treatments were imposed separately on these two collections of $\mathbf{4 0}$ slides each. In Procedure 1, only the slides from the first half-tray were used, and, for all these slides, whether or not it contained a dot, the curve's interior was bright red, vividly visible to humans and, presumably, to pigeons. In Procedure 2, slides from both half-trays were used: The first half continued with bright red interiors, whereas those from the second half-tray had dark red interiors, only just visible to the experimenters as distinct from the black background. In Procedure 3, slides from both half-trays were used: Slides from the first half now had curves with black interiors, physically the same as the black exterior, whereas those from the second half-tray continued to have dark red interiors. In Procedure 4, only first half-tray slides were used, and they continued unchanged with the black interiors. In Procedure 5, slides from both half-trays were used: Slides from the first half-tray were the same as in Procedures 3 and 4 , continuing to have black interiors; slides from the second half-tray had black interiors and the dots were in new locations. Note that the slides in the second half-tray in Procedure 5 constituted a transfer test for insideness, inasmuch as they were all slides with dots in locations never before shown to the subjects. Additional transfer tests were conducted in Phase 2, to be described below.

Results from Phase 1 are plotted in Figure 2, in separate averages for the pigeons for whom dot-inside was positive (i.e., Pigeons 1 and 2 ) and for whom dot-outside was positive (i.e., Pigeons 3 and 4). Behavior is measured by the ratio of positive to overall responding, $P /(P+N)$. From Sessions 53 to 56 (Procedure 1, Table 2), only slides from the first half-tray were used (filled points, solid lines), and the interiors were bright red. Evidence of discrimination rapidly appeared. On Session 57 , slides from

Table 2

Phase 1 Procedures

\begin{tabular}{|c|c|c|c|}
\hline Procedure & Interior of Curve & Sessions & $\begin{array}{c}\text { Session } \\
\text { Numbers }\end{array}$ \\
\hline 1 & All bright red & 4 & $53-56$ \\
\hline 2 & $\begin{array}{l}\text { Half bright red } \\
\text { Half dark red }\end{array}$ & 14 & $57-70$ \\
\hline 3 & $\begin{array}{l}\text { Half dark red } \\
\text { Half black }\end{array}$ & 12 & $71-82$ \\
\hline 4 & $\begin{array}{l}\text { All black, } \\
\text { original slides }\end{array}$ & 45 & $83-127$ \\
\hline 5 & $\begin{array}{l}\text { All black, } \\
\text { new slides }\end{array}$ & 92 & $128-218$ \\
\hline
\end{tabular}




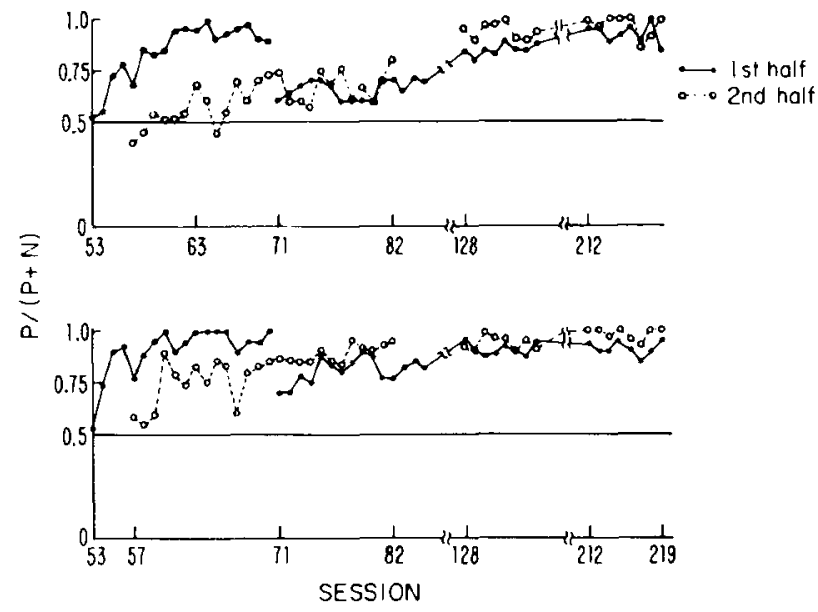

Figure 2. Proportion of responding to pasitive stimuli with respect to total responding during the five procedures of Phase 1 (see text and Table 2). Solid points are for slides in the first half of the projector tray; open points, for slides in the second half; but slides from all occupied positions in the tray were sampled in random order during sessions. Pigeons 1 and 2 (dot-inside positive) were averaged for the upper graphs; Pigeons 3 and 4 (dot-outside positive), for the lower graphs.

the second half-tray were also used (open points, dashed lines), and, for these, the interiors were dark red (Procedure 2). There was no sign of transfer of discrimination to the dark red interiors, but both groups of pigeons soon began to discriminate these positives and negatives as well. The discrimination of insideness for the dark red interiors proceeded less rapidly than, and perhaps to a less high level as, that for bright red interiors.

On Session 71, the interiors of the curves for the first half-tray were made the same black as the exterior (Procedure 3), so that at this point the only basis of discrimination should have been insideness (setting aside for now the possibility that the subjects learned the geometric configurations of dots and curves when the interiors were bright red, which they recognized when the interiors were black). Both groups suffered a substantial, but clearly not total, drop in discrimination with this change. This evidence of transfer is the first sign, albeit an arguable one, of discrimination based on insideness. The two groups of subjects apparently did not differ systematically in the amount of transfer. Slides in the first half-tray had no further changes in Phase 1, which is to say, until Session 219.

From Sessions 82 to 127 , only first half-tray slides were used (Procedure 4), and the discrimination between positives and negatives for these black interiors improved gradually. For the first group, the level reached a discrimination ratio in the mid-.80s; for the second group, somewhat higher. On Session 128, new slides were used for the second half-tray (Procedure 5): The interiors were black, and the curves were the same as previously, but the dots were in locations never seen before by the subjects. All pigeons immediately transferred the discrimi- nation to these new stimuli. Indeed, on almost every session, the level of discrimination for the new slides in the second half-tray was higher than that for those in the first half-tray, which had been seen for over $\mathbf{5 0}$ sessions. Later, we will discuss why this test might have resulted in "supergeneralization."

\section{Phase 2}

This phase comprised a series of procedural probes designed to examine the generality, and the stimulus characteristics, of the pigeon's rule for insideness. To determine whether it was the presence or absence of dots that controlled responding, on Session 220 , immediately following the final point in Figure 2, 10 of the slides were replaced with 10 new ones, similar to those removed except that 6 of the new slides had no dots at all and 4 of them had two dots, one inside the curve and the other outside. No reinforcements could be earned for the test slides. Each pigeon's average number of pecks per 10-sec exposure to the test slides is given in Table 3 .

For all subjects, two dots produced more responding than did no dots. The difference in average rates in Table 3 understates how large the effect actually was. On 23 of the 24 occasions when a pigeon saw a dotless slide (i.e., 4 pigeons $\times 6$ slides), there were no pecks at all. On 14 of the 16 occasions when a pigeon saw a slide with two dots (i.e., 4 pigeons $\times 4$ slides), there was pecking. Whether a pigeon was pecking for dot-inside or dotoutside apparently made no difference in the control exerted over behavior by dots.

The final two columns in Table 3 give average pecks per $10 \mathrm{sec}$ for the 70 ordinary stimuli for this session, separated into positives and negatives. The rates of responding have been averaged across subjects to show the similarity of rates of responding to positives and twodot stimuli, and to negatives and no-dot stimuli. The similarity suggests that the pigeons pecked when they saw a dot in the positive location (i.e., inside for Pigeons 1 and 2, outside for Pigeons 3 and 4), without regard to other aspects of the stimuli. Dots in the negative location, as for the two-dot stimuli, did not suppress responding; the absence of a dot in the negative location, as for the no-dot stimuli, did not facilitate responding.

The no- and two-dot slides were then removed, and on each of the three following sessions, 10 slides were replaced with 10 new ones, 5 dot-inside and 5 dot-outside,

Table 3

Each Pigeon's Average Number of Pecks per 10-sec Stimuli

\begin{tabular}{ccccc}
\hline Subject & No Dot & Two Dots & Negative & Positive \\
\hline \multicolumn{5}{c}{ Dot-Inside } \\
1 & 0 & 3.5 & .06 & 3.6 \\
2 & 1.2 & 3.3 & .14 & 4.7 \\
& \multicolumn{5}{c}{ Dot-Outside } & & \\
3 & 0 & 8.5 & .26 & 7.9 \\
4 & 0 & 5.3 & .83 & 8.5 \\
Mean & .30 & 5.15 & .32 & 6.18 \\
\hline
\end{tabular}




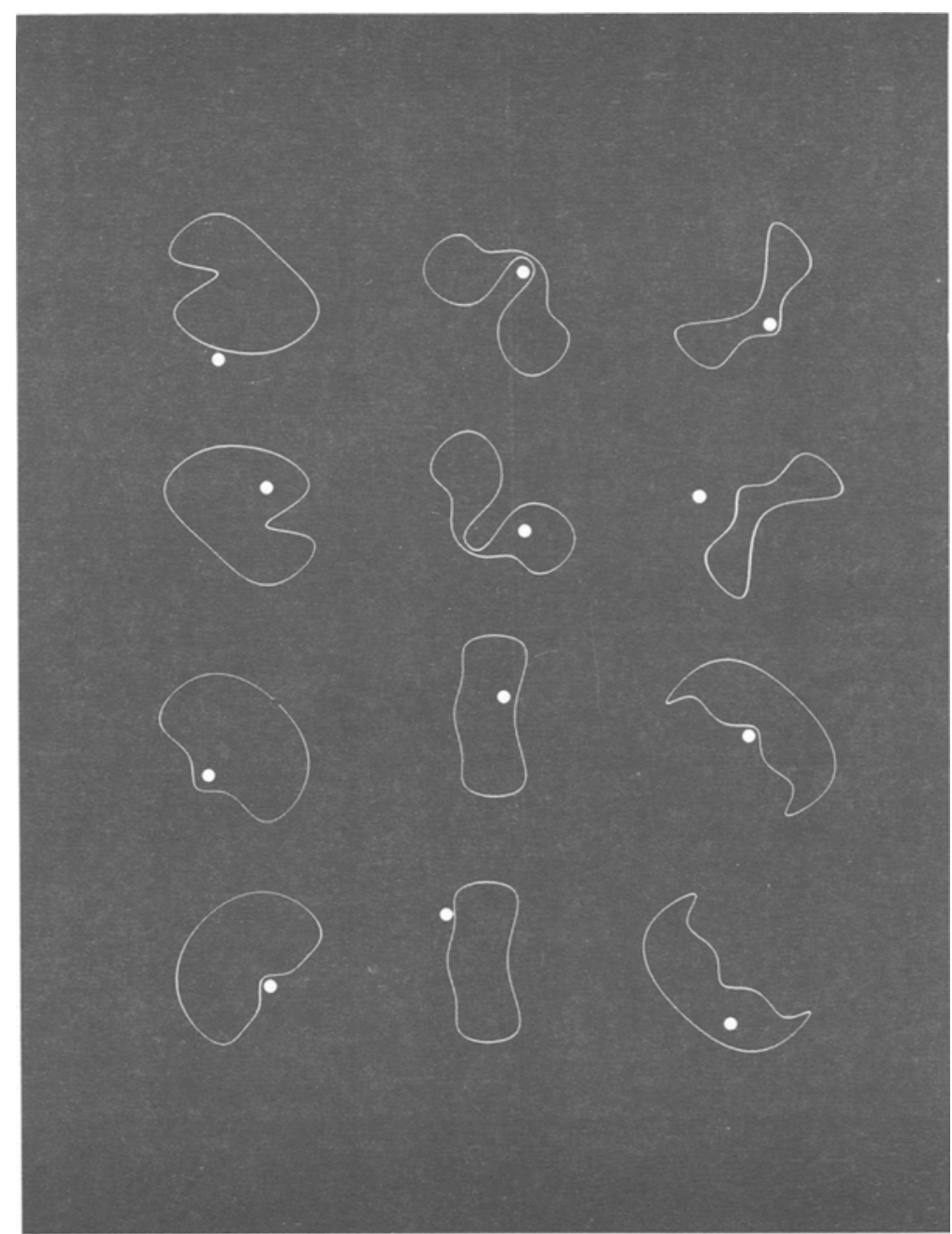

Figure 3. Twelve of the $\mathbf{3 0}$ stimuli used to test transfer of the insideness concept. Shapes and dot locations were all novel.

and with new curves of different shape, as illustrated in Figure 3. Every test stimulus was different and the dots were in various locations. Reinforcement was presented for positives, and not for negatives. The bar graphs in Figure 4 summarize the results of these 12 generalization tests (i.e., 4 pigeons $\times 3$ sets of 10 slides). The responding shown here occurred prior to any reinforcement in the presence of each test stimulus, enabling us to assess generalization from the training stimuli.

With no exceptions, for every set of 10 new slides, responding to the positives exceeded responding to the negatives, indicating a generalized control over responding by insideness. From Figure 2, we can estimate that the discrimination ratio, $P /(P+N)$, was over .9 for all subjects at this stage in the experiment with the stimuli used in training. For the test stimuli represented in Figures 3 and 4, this ratio was lower in all cases: Summing over the three generalization tests, for Pigeon 1, it was .79; for Pigeon 2, .80; for Pigeon 3, .68; and for Pigeon 4,
.80. The insideness rule was generalized with some decrement to new curves.

\section{Discriminability}

The results in Phases 1 and 2 meet the usual criteria of a generalized concept (in this instance, a rule for insideness). All pigeons successfully discriminated exemplars of insideness with hoth dots and curves seen for the first time. Previous research of this sort has, however, indicated that a pigeon's generalized concept is often contaminated by specific features of stimuli used in training, as if it were not fully under the control of the stimulus class at the appropriate level of abstractness (see Greene, 1983; Herrnstein, in press; Pisacreta, Redwood, \& Witt, 1984). We examined the present results for a similar contamination by lower level features of the stimuli.

Ten sessions during the final procedure of Phase 1 (Sessions 205-214, see Figure 2), when the interiors of all curves were black, were used for this analysis of dis- 


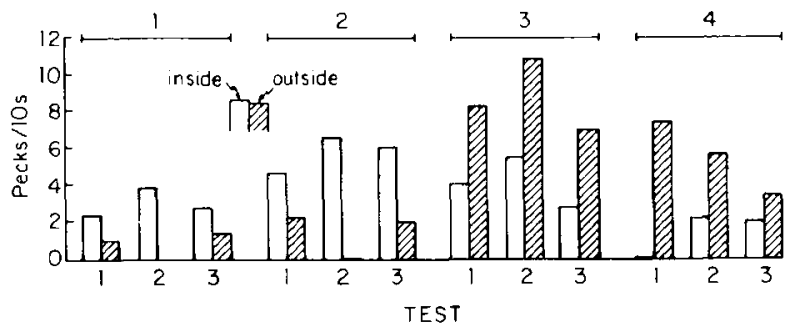

Figure 4. Pecks per 10 sec for each of the 4 subjects, responding to the $\mathbf{3 0}$ new stimuli used to test transfer. For Pigeons 1 and 2, dotinside was positive; for Pigeons 3 and 4 , it was negative. Ten of the stimuli were introduced on each of three consecutive sessions, and the results are summarized by separate pairs of bar graphs for each session. Responding is prior to the first reinforcement received in the presence of each novel positive stimulus.

criminability. Consider the stimuli as illustrated in Figure 1. Presumably, if the problem of discriminating insideness as such competes with discriminating based on local cues, then the most difficult stimuli should be those in which the dot was within the bulge on, or the bay in, the curve, as the case may be. This should be so whether the pigeon was reinforced for responding to dot-inside or -outside, because, either way, the local cues around the dot would be most ambiguous when the dot is surrounded by the bay or bulge. For these exemplars, the local cues are, in fact, the same whether the dot is inside or outside and can be interpreted only after determining whether the curve has a bulge or a bay, a discrimination that is not obviously local. There were eight such stimuli per session for each pigeon, four positive and four negative, and the relevant results are presented in Table 4.

The first two columns present the rates of pecking per $10 \mathrm{sec}$ for these potentially ambiguous positive and negative stimuli. Pecking for the 10 sessions has been averaged for each stimulus, and the rates shown in Table 4 are medians over the four relevant stimuli for each entry. In all cases, the pigeons discriminated sharply between positive and negative stimuli, even though the local cues were ambiguous. The next two columns show that, on the whole, although these stimuli were discriminable despite the local ambiguity, there is some evidence of difficulty.

The averaged rates of pecking have been ranked within categories. That is, responding to the 40 positive stimuli

Table 4

Results from Discriminating Ambiguous Stimuli

\begin{tabular}{|c|c|c|c|c|c|c|}
\hline \multirow[b]{3}{*}{ Subject } & \multirow{2}{*}{\multicolumn{2}{|c|}{ Pecks $/ 10 \mathrm{sec}$}} & \multicolumn{4}{|c|}{ Mean Rank } \\
\hline & & & \multicolumn{2}{|c|}{ Within Category } & \multicolumn{2}{|c|}{ Overall } \\
\hline & Positive & Negative & Positive & Negative & Positive & Negative \\
\hline \multicolumn{7}{|c|}{ Dot-Inside } \\
\hline 1 & 3.30 & 1.7 & 11.0 & 4.25 & 11.5 & 36.8 \\
\hline 2 & 5.50 & .85 & 23.4 & 18.8 & 24.3 & 54.8 \\
\hline \multicolumn{7}{|c|}{ Dot-Outside } \\
\hline 3 & 11.3 & 4.4 & 12.5 & 2.5 & 13.0 & 42.5 \\
\hline 4 & 7.35 & 1.7 & 6.8 & 2.5 & 7.0 & 42.3 \\
\hline
\end{tabular}

were ranked, as were responses to the $\mathbf{4 0}$ negative stimuli. A high rank (i.e., close to 1) represents a high rate of pecking, relative to pecking to other stimuli within the stimulus category. For the positive category, a high rank represents an easy stimulus, and a low rank (i.e., close to 40) represents a difficult stimulus. For the negative category, the reverse is true: A high rank represents a difficult stimulus (because responding to negative stimuli is erroneous), and a low rank represents an easy stimulus. For either category, average difficulty predicts ranks averaging 20.5 .

The central two columns of Table 4 give the mean ranks for the four stimuli, so that the highest possible entry is 2.5 and the lowest possible is 38.5 . The average rank in either positive or negative category is, of necessity, 20.5 . For averages of four stimuli, the confidence interval (99\%) around 20.5 , assuming random assignment of ranks, extends from 8 to 33. Pigeons 3 and 4 have entries of 2.5 for negative stimuli, which means they had difficulty when a dot was inside the curve and inside a bulge. Pigeon 1 has an entry of 4.25 for the negative stimuli, which means it had difficulty when a dot was outside the curve and inside a bay. For these 3 pigeons, negative stimuli with dots in the bay or bulge were significantly more difficult than were the average negative stimulus, but, as the first two columns show, were still being discriminated from positive stimuli with dots in the bay or the bulge. Pigeon 2 had the least difficulty with the ambiguous negative stimuli. For no pigeon was the dot in the bay or bulge a source of significant difficulty for positive stimuli. Indeed, for Pigeon 4, these stimuli were significantly easier, which means that it responded more to stimuli with dots outside when the dot was in a bay.

The final two columns of Table 4 give the mean ranks for the ambiguous stimuli when all $\mathbf{8 0}$ slides are ranked together. Reflecting the difference in rates of pecking in the first two columns of the table, the difference in rank for positive and negative stimuli confirm the presence of discrimination between the ambiguous stimuli for all subjects. We do not attempt to estimate the statistical significance of this rank difference, inasmuch as for every pigeon, every positive ambiguous stimulus was ranked above every negative ambiguous stimulus.

The test in Table 4 is based on a 10-session interval after considerable training with a particular set of 80 slides. At the end of Phase 2, we inserted 30 new slides (10 per session) and found that the insideness rule generalized with some decrement (see Figure 4). Of those 30 new slides, 10 were ambiguous in the present sensewith a dot inside either a bay or bulge. Five of these ambiguous stimuli can be seen in Figure 3: the second and fourth in the first column, the last in the second column, and the first and second in the third column. An analysis of these ambiguous slides alone shows that generalization failed for 3 of the 4 pigeons (discrimination ratios of $.54, .49$, and .46 for Pigeons 1,3 , and 4 , respectively). Only Pigeon 2 gave evidence, albeit weak evidence, of 
a rule for insideness generalizing to these stimuli-its discrimination index was .63 , whereas the index was .80 for the 30 stimuli as a whole. As noted earlier, these ambiguous, or difficult, stimuli were ones in which the dot was close to a sharp convexity or concavity, but this is not a local feature in the ordinary sense, for the controlling variable is precisely whether it is convexity or a concavity, and that information is not locally defined. For the $\mathbf{3 0}$ test stimuli as a whole, all pigeons generalized the insideness rule, as shown in Figure 4.

\section{DISCUSSION}

The pigeons were not spontaneously able to solve the problem of insideness, as posed by a set of 80 exemplars of simple curves with a single dot either inside or outside the curve. They were unable, over several dozen sessions, to learn to sort the $\mathbf{8 0}$ exemplars into two arbitrary categories of 40 , even though they are able to learn arbitrary sorts of even larger numbers of slides of natural scenes (e.g., Herrnstein \& de Villiers, 1980). An obvious hypothesis concerning the difficulty the pigeons had with these stimuli and with the task they were being asked to do is the unnaturalness of both.

Simple curves and dots are not things a pigeon can be expected to have sharply identified in its ordinary experience. And insideness per se, as an abstract relation, does not seem to be one that an animal in nature is likely to be called upon to use. In that respect, it may differ from such relations as "above" or "the same as." However, while insideness may be unnatural, essential components of the relation seem commonplace. The ability to segregate the visual field into distinct figures on a background is vital in nature. Moreover, although some figures stand out by clearly contrasting color or texture, others may be obscured (e.g., by shadows or nearer objects like branches). In such cases, some processing is required to see the figure integrally. In the extreme, only the outline of an object may be visible, and the animal would then have to find the interior of the outline by some sort of mental coloring processing (as was mentioned in the Introduction) or its analogue.

There are, then, speculative reasons from nature for supposing that a pigeon can identify the interior of a closed curve, such as those used in the present experiment. To see that a dot falls on this interior requires an additional processing step. In nature, this additional relationship sometimes corresponds geometrically to the interposition of one object in front of another, a cue to relative distance in the third dimension. It is likely that pigeons use the cue of interposition in the perception of distance. A dot falling on a region also corresponds in nature to a larger object being marked by a small region or spot on its surface. Pigeons probably rely on such surface markings in recognition of, for example, conspecifics.

Thus, the "painting" of a curve's interior may alreaoy have been present in the pigeon's visual system, part of its normal activity of seeing distinct entities in the visual field; likewise insideness, to the extent that it corresponds to three-dimensional interposition or to surface markings. Thus, every part of the relation of insideness has a plausible natural use, but what seems to be missing in nature is the abstract relation of insideness per se. Insideness is a rule defined geometrically in the present experiment on a two-dimensional surface. ${ }^{1}$ Generalizing natural experience, the pigeons might conceivably have initially seen a dot-inside stimulus as one in which the region bounded by the curve was one object and the dot was either a second, closer object or a marking on the first object, but nothing in three-dimensional nature seems to have equipped them to see it as the dot being two-dimensionally inside the curve.

The pigeons not only failed to learn the insideness rule spontaneously, their eventual learning of it fell short of complete generalizability or logical coherence. In generalization tests using two dots or none, they proved to be under the primary control of the dot, looking to see if it was inside or outside the curve, rather than the logically complementary condition, given the training stimuli, namely, looking to see if there was no dot outside or no dot inside. This appears to be a novel example of the often noted feature-positive effect, the finding that subjects find it easier to associate the positive class with the presence of a distinctive feature than they do with its absence (e.g., Jenkins \& Sainsbury, 1970). In the present experiment, the dot apparently served as the distinctive feature, whether the positive class was dot-inside or -outside.

The pigeons successfully generalized on first viewing to new dot locations if the curves were familiar and to some new curves and new dot locations. These results show unambiguously a degree of control by the abstract relation of insideness. But the pigeons had difficulty mainly when the dot fell within the bays or bulges that most severely test the abstract aspect of the relation. In discussing Figure 2, we noted that, in Procedure 5, the pigeons responded at a higher level of discrimination when they were tested with new stimuli than they had achieved with the original set. The reason is probably that the original set contained eight difficult cases, but the new stimuli, with dots in new locations, contained none.

It may be that with further exposure to varying shapes of regions, the pigeons would have overcome their troubles and learned to extend the insideness rule to the curves they had difficulty with and other more complex curves, but the present data do not quite live up to the ideal of a pure, abstract relational rule. Many demonstrations of abstract relational learning by pigeons and other subhuman animals are similarly contaminated by the specific features of the stimuli used in training (see Hermstein, 1985 , in press, for review and discussion of this issue). The neat distinction between bottom-up description and top-down, description-free predication that we discussed in the Introduction tends to be smeared in data from animal categorization. Control by an abstract relation tends not 
to be the all-or-none phenomenon it is depicted as in theoretical writings, but more like a point on a continuum, with greater or lesser degrees of confounding with descriptive particulars of the stimuli being discriminated.

However, it may be that pure, abstract relations are generally more a theoretical possibility than an empirical one. Ullman (1984) points out that the human capacity to detect insideness is far from unlimited. A sufficiently complex closed figure, with many convolutions, defies a human's ability to see whether a dot is inside it or not. Similarly, our pigeons had difficulty with new exemplars in which the dot was surrounded by ambiguous local cues. For familiar exemplars, they had no such difficulty. The generalization probably failed not because the pigeons were unable to deal, in some simple processing sense, with the convolutions of the test figures, for they do not seem more complex in that respect than do the training stimuli (see Figures 1 and 3). Their problem, rather, was probably that the test shapes were too different from what they had been trained with.

Given the present results, as well as many others, it seems that a natural system often (if not always) generalizes conservatively rather than purely abstractly-if the stimuli differ in particular new ways from the stimuli the organism has been trained with, the rule for categorization is suspended. Such a mechanism may have adaptive benefits in environments in which objective variation is unpredictable and unbalanced. Inasmuch as nature rarely, if ever, assigns categories of reinforcement to descriptionfree abstract relations, it should be no surprise that organisms do not readily generalize abstract relations beyond the bounds of "common" variation, whatever that qualification proves to entail. Our experiment was not designed to uncover which dimensions of variation disrupt or delimit the generalization of an abstract relation, but knowing more about this could further illuminate the nature of the controlling rule for whatever generalization is observed.

We were able to enhance the pigeons' discriminations between dot-inside and dot-outside stimuli by a literal application of Ullman's notion of a visual routine that paints the inside of figures as the top-down computation that enables a human observer to use the insideness rule. Other visual routines may likewise have dictated coloring the insides of the curves as the appropriate training procedure. In any case, we may have been drawing on an existing capacity in the pigeon, essential for the visual segregation of objects in nature. The main implication of the present experiment is that subjects can be taught to use a relational rule that is not spontaneously accessible, but perhaps only if the component stimulus analyses are already present in the organism (as we suggested above, they probably were in this instance). It is as though the pigeon needs to be "told," by the earlier colored slides, to pay attention to the inside of the curve as a component of the stimulus. Of course, such reasoning is not only speculative, but vague-we cannot yet specify which stimulus analyses must be present in order to build up some degree of abstract relational discrimination.

Even so, the results teach one clear lesson: Questions of cognitive capacity need to be more sharply posed than they usually are. The question is not whether pigeons (or any other system) can use insideness as a rule for categorization, but under what conditions they do or do not. The initial results of the experiment, when all pigeons were failing to discriminate, could have been interpreted as an incapacity to use the rule, but the later results suggest at least some revision of that interpretation. Given teachability, the difference between what is being done by a system and what it is capable of doing may be decisive for any theoretical or practical purpose. Inasmuch as a pigeon needs to be programmed to use insideness, just as a computer must be, it may be a more convenient subject than a human being for discovering how this visual routine might be simulated by a vision machine.

\section{REFERENCES}

Commons, M. L., Herrnstein, R. J., \& W AGner, A. R. (Eds.). (1983). Quantitative analyses of behavior: Discrimination processes (Vol. 4). Cambridge, MA: Ballinger.

GrEeNE, S. L. (1983). Feature memorization in pigeon concept formation. In M. L. Commons, R. J. Herrnstein, \& A. R. Wagner (Eds.), Quantitative analyses of behavior: Discrimination processes (Vol. 4, pp. 209-228). Cambridge, MA: Ballinger.

Herrnstein, R. J. (1984). Objects, categories, and discriminative stimuli. In H. L. Roitblat, T. G. Bever, \& H. S. Terrace (Eds.), Animal cognition (pp. 233-261). Hillsdale, NJ: Erlbaum.

Herrnstein, R. J. (1985). Riddles of natural categorization. Philosophical Transactions of the Royal Society, London, B308, 129-144.

HerRnSteIn, R. J. (in press). Levels of categorization. In G. M. Edelman, W. E. Gall, \& W. M. Cowan (Eds.), Signal and sense: Local and global order in perceptual maps. New York: Wiley.

Herrestein, R. J., \& DE VIlliers, P. A. (1980). Fish as a natural category for people and pigeons. In G. H. Bower (Ed.), The psychology of learning and motivation (Vol. 14, pp. 59-95). New York: Academic Press.

Jenkins, H. M., \& SAINSbury, R. S. (1970). Discrimination learning with the distinctive feature on positive or negative trials. In D. 1 . Mostofsky (Ed.), Attention: Contemporary theory and analysis (pp. 239-273). New York: Appleton-Century-Crofts.

LiNDSAY, P. H., NoRMAN, D. A. (1977). Human information processing: An introduction to psychology (2nd ed.). New York: Academic Press.

Mackintosh, N. J. (1983). Conditioning and associative leaming. New York: Oxford University Press.

MARR, D. (1976). Early processing of visual information. Philosophical Transactions of the Royal Society, London, B275, 483-524.

Minsky, M., PAPERT, S. (1969). Perceptrons: An introduction to computational geometry. Cambridge, MA: MIT Press.

Pisacreta, R., Lefave, P., Lesneski, T., \& Potter, C. (1985). Transfer of oddity learning in the pigeon. Animal Learning \& Behavior, $13,403-414$.

Pisacreta, R., Redwood, E., \& WITt, K. (1984). Transfer of matchingto-figure samples in the pigeon. Joumal of the Experimental Analysis of Behavior, 42, 223-237.

Ullman, S. (1984). Visual routines. Cognition, 18, 97-159.

URCuIOLI, P. J. (1985). On the role of differential sample behaviors in matching-to-sample. Journal of Experimental Psychology: Animal Behavior Processes, 11, 502-519. 
Vaughan, W., JR., \& Greene, S. L. (1984). Pigeon visual memory capacity. Journal of Experimental Psychology: Animal Behavior Processes, 10, 256-271.

Zentall, T. R., \& Hogan, D. E. (1978). Same/different concept learning in the pigeon: The effect of negative instances and prior adaptation to transfer stimuli. Journal of the Experimental Analysis of Behavior, 30, 177-186.
NOTE

1. It should be noted that insideness on a plane is a different matter entirely from the inclusion of one object within another in a threedimensional space. Two-dimensional insideness has more in common with three-dimensional interposition than with three-dimensional inclusion.

(Manuscript received January 4, 1988;

revision accepted for publication January 3, 1989.) 